\title{
Corticohippocampal Contributions to Spatial and Contextual Learning
}

\author{
Rebecca D. Burwell, Michael P. Saddoris, David J. Bucci, and Kjesten A. Wiig \\ Departments of Psychology and Neuroscience, Brown University, Providence, Rhode Island 02912
}

Spatial and contextual learning are considered to be dependent on the hippocampus, but the extent to which other structures in the medial temporal lobe memory system support these functions is not well understood. This study examined the effects of individual and combined lesions of the perirhinal, postrhinal, and entorhinal cortices on spatial and contextual learning. Lesioned subjects were consistently impaired on measures of contextual fear learning and consistently unimpaired on spatial learning in the Morris water maze. Neurotoxic lesions of perirhinal or postrhinal cortex that were previously shown to impair contextual fear conditioning (Bucci et al., 2000) or contextual discrimination (Bucci et al., 2002) caused little or no impairment in place learning and incidental learning in the water maze. Combined lesions of perirhinal plus lateral entorhinal or postrhinal plus medial entorhinal cortices resulted in deficits in acquisition of contextual discrimination but had no effect on place learning in the water maze. Finally, a parahippocampal lesion comprising combined neurotoxic damage to perirhinal, postrhinal, and entorhinal cortices resulted in profound impairment in acquisition of a standard passive avoidance task but failed to impair place learning. In the same experiment, rats with hippocampal lesions were impaired in spatial navigation. These results indicate that tasks requiring the association between context and an aversive stimulus depend on corticohippocampal circuitry, whereas place learning in the water maze can be accomplished without the full complement of highly processed information from the cortical regions surrounding the hippocampus. The evidence that different brain systems underlie spatial navigation and contextual learning has implications for research on memory when parahippocampal regions are involved.

Key words: perirhinal; postrhinal; entorhinal; parahippocampal; hippocampal; water maze; fear conditioning; passive avoidance

\section{Introduction}

The role of corticohippocampal circuitry in mnemonic function is an important topic in research investigating medial temporal lobe structures and memory. The perirhinal (PER) and postrhinal (POR) cortices provide the major source of neocortical sensory association information to the entorhinal cortex (EC), the origin of the perforant path projection to the dentate gyrus and CA3 regions of the hippocampus. Neuroanatomical studies define two relatively separate corticohippocampal processing streams involving the perirhinal and postrhinal cortices. These processing streams include direct connections with the hippocampus and indirect connections through the entorhinal cortex (Kosel et al., 1983; Naber et al., 1997; Shi and Cassell, 1999). Perirhinal and postrhinal connectivity with the entorhinal cortex is segregated such that perirhinal cortex projects preferentially to

\footnotetext{
Received Dec. 17, 2003; revised March 5, 2004; accepted March 5, 2004.

This research was supported by a National Science Foundation career award (IBN 9875792) to R.D.B., a National Institute of Mental Health postdoctoral fellowship (F32-MH12426) to D.J.B., and a Howard Hughes Medical Institute fellowship to K.A.W.

Correspondence should be addressed to Dr. Rebecca D. Burwell, Department of Psychology, Brown University, 89 Waterman Street, Providence, RI 02912. E-mail: rebecca_burwell@brown.edu.

M. P. Saddoris's present address: Department of Psychology, Johns Hopkins University, 3400 North Charles Street, Baltimore, MD 21218.

D. J. Bucci's present address: Department of Psychology, University of Vermont, 2 Colchester Avenue, Burlington, VT 05405.

K. A. Wiig's present address: Sention, Inc., 1 Richmond Square, Providence, RI 02906.

D0I:10.1523/JNEUROSCI.0410-04.2004

Copyright $\odot 2004$ Society for Neuroscience $\quad$ 0270-6474/04/243826-11\$15.00/0
}

the lateral entorhinal area (LEA) and postrhinal cortex projects preferentially to the medial entorhinal area (MEA) (Burwell and Amaral, 1998a).

The hippocampus has been implicated in both spatial and contextual learning. The Morris water maze task, a well established test of spatial navigation, is sensitive to hippocampal (HC) (Morris et al., 1982) and fornix damage (Devan and White, 1999; Pouzet et al., 1999). Hippocampal and fornix damage also disrupt contextual fear conditioning (for review, see Maren et al., 1998) (Phillips and LeDoux, 1995; Maren and Fanselow, 1997; Bannerman et al., 2001). After a careful review of the available evidence, however, Holland and Bouton (1999) concluded that the hippocampus is involved in some, but not all, aspects of contextual learning (Young et al., 1994; Maren et al., 1997; McNish et al., 2000).

Previous studies of the perirhinal, postrhinal, and entorhinal cortices have yielded variable results on spatial learning and navigation tasks (for review, see Aggleton et al., 2000). Some studies report impairment on the water maze task (Kaut and Bunsey, 2001; Liu and Bilkey, 2001) and others do not (Bussey et al., 1999; Mumby and Glenn, 2000). A radial maze study suggested that impairment associated with perirhinal-postrhinal damage might depend more on the type of memory tested than on the spatial requirements of the task (Jarrard et al., 2004).

The perirhinal and postrhinal cortices are also implicated in contextual fear learning tasks, including contextual discrimination (Bucci et al., 2002), passive avoidance (Tassoni et al., 1999), 
and contextual fear conditioning (Corodimas and LeDoux, 1995; Sacchetti et al., 1999; Bucci et al., 2000; but see Phillips and LeDoux, 1995; Herzog and Otto, 1997). Such tasks are thought to require the processing of spatial information about the environment. One possibility is that spatial tasks vary in the degree to which they require higher-order processing of polymodal sensory information. Thus, spatial and contextual tasks may differ in the brain systems that are obligatory for normal function.

The present study examined the contribution of perirhinal, postrhinal, and entorhinal cortices to spatial and contextual learning in the same animals. In experiments 1 and 2, rats with neurotoxic lesions of perirhinal or postrhinal cortex that were previously shown to be impaired on contextual fear tasks (Bucci et al., 2000, 2002) were tested in standard place learning and an incidental learning task in the water maze. In experiments 3 and 4 , rats with larger parahippocampal $(\mathrm{PH})$ lesions including entorhinal cortex were tested in place learning in the water maze and on a contextual fear task, either contextual discrimination or passive avoidance, another task thought to test associative learning to a context (Stubley-Weatherly et al., 1996). If spatial and contextual learning are similarly supported by the cortical regions that provide the primary sensory association input to the hippocampus, then damage to these regions should cause deficits on both spatial navigation and associative-contextual tasks.

\section{Materials and Methods}

\section{Experiment 1: effects of PER and POR lesions on place learning in} the water maze

Subjects. Subjects for the water maze were 37 male Long-Evans rats (Charles River Laboratories, Wilmington, MA) that had been used previously in a study of the effects of perirhinal and postrhinal neurotoxic lesions on the acquisition and retention of contextual fear conditioning (Bucci et al., 2000). Rats weighed $\sim 350$ gm at the time of surgery. On arrival in the vivarium, rats were housed in groups of three for 1 week and maintained on a $12 \mathrm{hr}$ light/dark cycle with ad libitum access to food and water. During that time, rats were handled daily for $\sim 1-2 \mathrm{~min}$. Rats were then separated and housed individually for the remainder of the experiment. Lesions were produced before testing on contextual fear conditioning. Training on the water maze was conducted 2 months after training on fear conditioning was completed.

Surgery. Anesthesia was induced with a 3\% halothane-oxygen mixture and maintained with $1-2.5 \%$ halothane. Rats were mounted in a stereotaxic apparatus (David Kopf Instruments, Tujunga, CA). Under aseptic conditions, the scalp was incised and retracted to the side, and the head was leveled between bregma and lambda. Small holes were drilled through the skull above the designated lesion sites. Bilateral neurotoxic lesions were made with ibotenic acid (10 mg/ml; Sigma, St. Louis, MO) dissolved in $0.1 \mathrm{M}$ phosphate buffer (PB). Ibotenic acid was pressure injected through a glass pipette $(50 \mu \mathrm{m}$ tip). For PER lesions $(n=10)$, injections of 25-50 $\mathrm{nl}$ were made at each of the following stereotaxic coordinates: $2.3,3.3,4.3,5.2,6.4$, and $7.1 \mathrm{~mm}$ posterior to bregma; 6.3 , 6.4, 6.5, 6.6, 6.8, and $6.2 \mathrm{~mm}$ lateral from the midline; and 7.4, 7.6, 7.7, $7.0,6.2$, and $5.2 \mathrm{~mm}$ below the skull surface, respectively. For POR lesions $(n=14)$, the pipette was angled at $22^{\circ}$ from vertical, with the tip oriented rostrally. Two injections were made. The first was $2.0 \mathrm{~mm}$ posterior to lambda, $0.3 \mathrm{~mm}$ lateral to the lateral ridge of the skull, and $5.92 \mathrm{~mm}$ ventral to the skull surface. The second was $2.0 \mathrm{~mm}$ rostral to lambda, 0.2 $\mathrm{mm}$ medial to the lateral ridge, and $4.85 \mathrm{~mm}$ ventral to the skull surface. All injections were made at a rate of $33 \mathrm{nl} / \mathrm{min}$. The micropipette was left in place for $30 \mathrm{sec}$ before and $120 \mathrm{sec}$ after each injection. For the sham surgery group (CTL) $(n=13)$, holes were drilled as above for either PER or POR lesions. A 2 week recovery period preceded behavioral training.

Behavioral procedures. Spatial learning was assessed using the Morris water maze (Morris, 1984). The maze included a circular tank $(180 \mathrm{~cm}$ in diameter) filled to $13 \mathrm{~cm}$ below the edge of the tank with $27^{\circ} \mathrm{C}$ water that was made opaque by the addition of white tempera paint. A circular escape platform (12 cm in diameter) was located $1 \mathrm{~cm}$ below the surface of the water in a constant location in the northwest quadrant of the tank, $43 \mathrm{~cm}$ from the wall. The maze was surrounded by white curtains, which were marked by four large stimuli varying in size, shape, and luminance. A video camera located above the center of the tank was interfaced with a videocassette recorder and an HVS Image Analysis tracking system. Data from all training and testing sessions were collected using HVS software (Water2020, HVS, Hampton, UK).

Rats were first acclimated to the maze during a one-trial habituation session. The platform was removed from the pool, and rats were allowed to swim for $60 \mathrm{sec}$ before being returned to the home cage. Place training began the day after habituation. The platform remained in a constant position throughout place training. Place training consisted of three trials each day for 8 consecutive days. On each trial, the rat was lowered by hand into the pool, facing the inside wall of the tank, at one of four pseudorandomly varied start positions that were spaced equally around the rim of the tank. If a rat did not reach the platform within $90 \mathrm{sec}$, it was guided to the platform by the experimenter. Rats remained on the platform for $15 \mathrm{sec}$ and then were returned to a holding cage for a $30 \mathrm{sec}$ intertrial interval (ITI). The third trial every other day (i.e., every sixth trial) was a $30 \mathrm{sec}$ probe trial during which the platform was removed. Thus, a probe trial was interpolated at the end of each block of five training trials for a total of four probe trials for the $8 \mathrm{~d}$ procedure. The interpolated probe trials, in which the hidden platform was removed, were used to evaluate search strategy. At the end of place training, performance of all rats was assessed for cue learning with a visible platform. The location of the visible platform and start location varied from trial to trial in a single session of six training trials.

Histology. Subjects were deeply anesthetized with sodium pentobarbital (Nembutal, $100 \mathrm{mg} / \mathrm{kg}$ ) and transcardially perfused with normal saline $(2 \mathrm{~min})$ followed by $4 \%$ paraformaldehyde $(20 \mathrm{~min})$ in $0.1 \mathrm{~m}$ sodium $\mathrm{PB}, \mathrm{pH}$ 7.4. Flow rate was calibrated at $35-40 \mathrm{ml} / \mathrm{min}$. After perfusion, each brain was removed from the skull and postfixed for $6 \mathrm{hr}$ at $4^{\circ} \mathrm{C}$ in buffered paraformaldehyde. Brains were then cryoprotected for 24-48 $\mathrm{hr}$ at $4^{\circ} \mathrm{C}$ in a solution of $20 \%$ glycerol in $0.1 \mathrm{M} \mathrm{PB}$. Coronal brain sections were cut at $40 \mu \mathrm{m}$ on a freezing microtome. Sections were collected in two series for POR-lesioned brains and four series for PER-lesioned brains for subsequent processing and storage. One series was collected in a $10 \%$ formalin solution in preparation for cell staining. That series was subsequently mounted and stained with thionin. The remaining series were collected and stored at $-20^{\circ} \mathrm{C}$ in cryoprotectant tissue-collecting solution consisting of $30 \%$ ethylene glycol and $20 \%$ glycerol in $0.1 \mathrm{M} \mathrm{PB}$.

Tissue damage was assessed using coronal sections at $240 \mu \mathrm{m}$ intervals for POR lesions and $480 \mu \mathrm{m}$ intervals for PER lesions. Camera lucida techniques were used to produce drawings that included regional borders and circumscribed the location of tissue damage. Tissue damage was identified by missing tissue, necrosis, or marked thinning of the cortex. For each coronal section, aerial measurements included the total area of the target region and the area of the target region that was damaged. Previous studies suggest that the extent of the lesion along the rostrocaudal axis is more predictive of efficacy of the lesion than total area (Bucci et al., 2000). Thus, we also assessed the proportion of sections in the rostrocaudal plane that exhibited damage. Subjects were retained in the experiment when a lesion involved extensive and distributed bilateral damage to the target region but did not include substantial bilateral damage to any region outside the target region.

Data analysis. Several measures were used to assess performance in the water maze. For place training trials, the primary measures for place training and cue training were latency and cumulative distance (Gallagher et al., 1993). The rat's proximity to (or distance from) the platform was sampled 10 times per second during a trial, and a mean was calculated for each second of the training trial. Cumulative distance was calculated as the sum of the $1 \mathrm{sec}$ means per training trial. For probe trials, the primary measure was average proximity to the platform. Average proximity was calculated as the average distance of the rat over the entire $30 \mathrm{sec}$ probe trial. Average swim speed was also calculated during place training.

Latency, cumulative distance, and swim speed were analyzed using repeated-measures ANOVA (rANOVA) with group (CTL, PER, POR) as the between-subject variable and block (average of five training trials) as 
the within-subject variable. Average proximity during the probe trials was analyzed using rANOVA with group and block as the between- and within-subject variables, respectively.

To address issues about correlation of performance across tasks, a single measure, a spatial learning index, was constructed from the average proximity measure for the four probe trials by summing weighted average proximities on the basis of archival performance of normal young rats (Gallagher et al., 1993). This spatial learning index was correlated with measures of contextual learning using the Pearson $r$ correlation coefficient.

An $\alpha$ level of 0.05 was adopted for all analyses, which were conducted with SAS version 8.0 (SAS Institute, Cary, NC).

\section{Experiment 2: effects of PER and POR lesions on place and incidental learning in the water maze}

Subjects. Subjects were 29 male Long-Evans rats (Charles River Laboratories) that were used previously in a study of the effects of perirhinal and postrhinal neurotoxic lesions on contextual fear discrimination (Bucci et al., 2002). Subjects weighed $\sim 400$ gm at the time of surgery. Otherwise, all housing and handling procedures were as described previously. Training in the Morris water maze was begun 6 weeks after training on contextual discrimination was completed.

Surgery and histology. Bilateral neurotoxic lesions of the POR $(n=8)$ or PER $(n=9)$ and sham lesions $(n=6)$ were made as reported previously (Bucci et al., 2002) and as described for experiment 1 , with one exception. For POR lesions, one injection was made at $22^{\circ}$ from lateral, $2.0 \mathrm{~mm}$ posterior to lambda, $0.3 \mathrm{~mm}$ lateral to the lateral ridge, and 6.14 $\mathrm{mm}$ ventral to the skull surface. Histology and lesion analysis were as described previously.

Behavioral procedures. Rats were trained in cue and place learning in the Morris water maze, and data were analyzed as described for experiment 1 . For cue and place training, the maze was surrounded by white curtains and complex stimuli as described previously. After a 3 week rest period, rats were trained in an incidental learning paradigm in the same water maze, but with different visual cues (Rapp et al., 1987). The maze was surrounded by dark curtains containing different visual stimuli, also of various sizes, shapes, and contrasting colors. Rats received two cuetraining trials per day ( $60 \mathrm{sec}$ ITI) for $6 \mathrm{~d}$. For each rat, the visible platform was located in the center of the same quadrant on each trial. The quadrant in which the platform was located was counterbalanced across animals. On day 7 , rats received one cue training trial as on previous days followed by a $90 \mathrm{sec}$ probe trial conducted during which the platform was removed from the maze. Measures analyzed for incidental learning were the same as those for place learning.

Experiment 3: effects of PER/LEA and POR/MEA lesions on place learning in the water maze and contextual discrimination

Subjects. Thirty-two male Long-Evans rats (Charles River Laboratories) weighing between 325 and 400 gm were used as subjects. Twenty-four rats received lesions or sham surgeries, and eight rats served as naive controls. All animals were housed and handled as described for experiment 1 . Each rat remained in the home cage for 2 weeks between contextual fear discrimination training and water maze training.

Surgery and histology. With the exception of lesion coordinates, all surgical procedures were the same as described previously. For PER/LEA lesions $(n=7)$, injections of $50 \mathrm{nl}$ per site were made at 2.3, 3.3, 4.3, 5.2, 6.4 , and $7.1 \mathrm{~mm}$ posterior to bregma, at $6.3,6.4,6.5,6.6,6.7$, and $6.1 \mathrm{~mm}$ lateral from the midline, and 8.0, 8.0, 8.0, 8.0, 7.1, and $6.2 \mathrm{~mm}$ below the surface of the skull, respectively. For the three most posterior sites, an additional injection of $50 \mathrm{nl}$ was at 7.0, 6.1, and $5.2 \mathrm{~mm}$ below the skull surface, respectively. For POR/MEA lesions $(n=9)$, the pipette was oriented $22^{\circ}$ rostrally, and an injection of $50 \mathrm{nl}$ per site was made at 2.0 $\mathrm{mm}$ posterior to lambda, $0.3 \mathrm{~mm}$ lateral from ridge, and $6.14 \mathrm{~mm}$ below skull surface. Another injection was made with the pipette oriented $10^{\circ}$ toward the midline, at $0.11 \mathrm{~mm}$ anterior to lambda, $3.7 \mathrm{~mm}$ lateral from midline, and $5.0 \mathrm{~mm}$ below skull surface ( $100 \mathrm{nl}$ per site). Sham animals (SHAM) $(n=6)$ received craniotomies as for PER/LEA- or POR/MEAlesioned rats, but no injections were made. Tissue preparation and analysis was as described for experiment 1 .
Behavioral apparatus. For contextual fear discrimination, four testing chambers $(30 \times 24 \times 27 \mathrm{~cm}$; MED Associates, St. Albans, VT) were used for all experiments. All chambers were composed of aluminum (two side panels) and Plexiglas (hinged front door, rear wall, and ceiling) and positioned in sound-attenuated cabinets in a well lit and isolated room. The floor of each chamber consisted of evenly spaced stainless steel rods attached to a shock generator and scrambler for the delivery of a foot shock. A centrally positioned house light located $1 \mathrm{~cm}$ from the ceiling in one of the side panels served to illuminate each chamber. Each testing chamber was provided with white noise $(70 \mathrm{~dB})$ from a speaker located on the right panel of the chamber.

Two of the four conditioning chambers on one side of the room ("Context A") contained identical sensory cues that distinguished them from the two chambers on the other side of the room ("Context B"). The specific cues that differed between the two contexts are described in detail below. For visual and tactile cues, Context A contained the cues present in the standard MED Associates chamber. The left aluminum panel of the testing chamber consisted of five identical evenly spaced nose poke holes measuring $2.5 \times 2.5 \mathrm{~cm}$ that were recessed $2 \mathrm{~cm}$ and located $2 \mathrm{~cm}$ above the floor grid. The right aluminum panel contained a standard food cup located in a recessed bay $(5 \times 5 \times 2 \mathrm{~cm})$ located equidistant from the edges of the panel and $2 \mathrm{~cm}$ above the floor grid. Additionally, three 2.5 $\mathrm{cm}$ circular panel lights (not used in this experiment) were located $5 \mathrm{~cm}$ apart on the right panel and separated by two vertical bars, $10 \mathrm{~mm}$ wide and $3 \mathrm{~mm}$ thick, that ran from the ceiling to the floor of the panel. For visual and tactile cues in Context $B$, laminated cue cards were placed over all walls. A white card with a black diamond $(23 \mathrm{~cm}$ across $)$ was placed over the left aluminum panel. A black card with three white circles $(6 \mathrm{~cm}$ diameter) arranged in a diagonal was placed over the right panel, leaving exposed only the house light. A plain white card covered the Plexiglas door. Resting between the rear Plexiglas wall and the back of the cabinet was a white cardboard panel with two, $5 \mathrm{~cm}$ vertically oriented maroon stripes. For olfactory cues, in each chamber a thin film of artificial flavoring in a 1:2 solution with water was sprayed into the bottom of the removable stainless steel pan below the floor grid. In Context A the odor was strawberry scented, and in Context B anise was used. The spatial arrangement of the shock and no-shock chambers in the testing room also differed. The time of day that rats received shocks remained constant throughout the testing procedure (i.e., shock was always delivered during the morning session). The apparatus for the Morris water maze experiment was the same as in experiment 1.

Behavioral procedures. The behavioral procedures for contextual fear discrimination were similar to previous experiments (Bucci et al., 2002). Rats received training in the test chambers for $3 \mathrm{~d}$. The third day was considered a test day. In the morning ( 9 A.M.), each rat was placed in a chamber containing a particular set of contextual cues for $6 \mathrm{~min}$. Rats received three footshocks ( $1.0 \mathrm{sec}, 0.1 \mathrm{~mA}$; 64 -sec inter-shock interval) beginning 3 min after being placed in the testing chamber ("shock context"). During the afternoon training session (4:30 P.M.), rats were exposed to another chamber with a different set of cues for $6 \mathrm{~min}$. No shock was delivered during the afternoon session ("no-shock context"). The procedures were counterbalanced such that some rats received shock in chamber A and other rats received shock in chamber B. The same temporal pattern of context exposure and shock delivery was maintained each day for each rat. Rats were immediately returned to their home cages after each session. Between sessions all surfaces of the chambers were cleaned with distilled water and a $0.5 \%$ solution of sodium hydroxide followed by a final rinse with distilled water. The Morris water maze task was conducted as described in experiment 1.

Behavioral observations. For contextual fear conditioning, each of the two daily sessions was divided into four $64 \mathrm{sec}$ epochs: three before receiving footshock (if delivered) and one after each shock delivery. Conditioning was assessed by measuring freezing behavior during the third epoch, which occurred immediately before any shocks (if in the shock condition) were delivered, as described previously (Bucci et al., 2002). Freezing (lack of movement other than respiration) is an associative fear response that is elicited by some aversive stimuli (Blanchard and Blanchard, 1969; Fanselow, 1980). Behavioral observations were made by a single experimenter, blind to experimental condition, every 8 sec during 


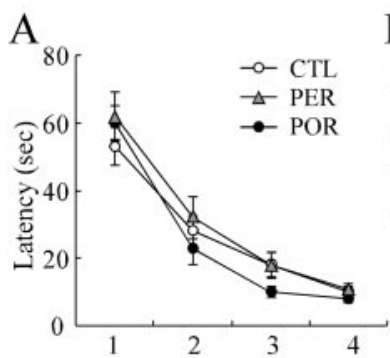

Blocks of Five Training Trials

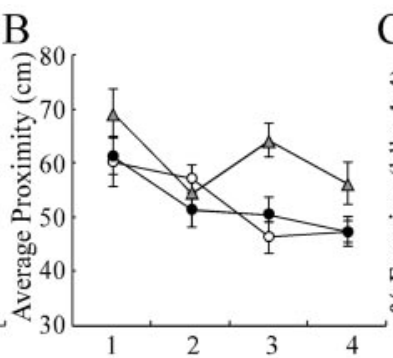

Probe Trials

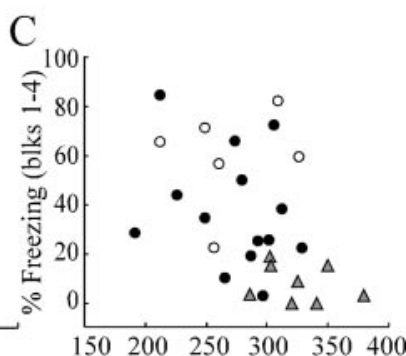

Watermaze Learning Index

Figure 1. Effects of damage to PER or POR in experiment 1. A, Latency to find the hidden platform during training trials. $B$, Average proximity to the platform during probe trials. Average proximity to the platform during probe trials was comparable across blocks for CTL and POR groups, but the PER group was mildly impaired. C, Relationship between mean contextual freezing in the first four 64 sec blocks of extinction (Bucci et al., 2000) and performance on the water maze (present study) as indicated by a learning index. Percentage of freezing during extinction in the contextual fear conditioning task was significantly correlated with performance on the water maze task overall, but not within groups. CTL, Control group; PER, perirhinal-lesioned group; POR, postrhinal-lesioned group. Data are mean \pm SEM.

the 64 sec block, yielding eight observations per epoch for each rat. The occurrence of freezing behavior was expressed as a percentage of total observations. A second rater, also blind to experimental condition, independently scored a subset of the sessions to assess inter-rater reliability. The results obtained by the two observers were significantly correlated $(p<0.01)$.

Data analysis. For contextual fear conditioning, freezing behavior was analyzed using rANOVA with group as a between-subjects variable and shock condition as a within-subjects variable. For some analyses, training day provided an additional within-subjects variable. Planned comparisons of significant main effects and interactions were assessed with appropriate contrasts. Analyses of performance on the Morris water maze were as described for experiment 1.

Experiment 4: effects of combined PER/POR/EC lesions on place learning in the water maze and passive avoidance learning

Subjects. Subjects for the water maze were 27 male Long-Evans rats (Charles River Laboratories) that weighed $\sim 350$ gm at the time of surgery. Animals were housed and handled as described for experiment 1 . Behavioral training on the water maze began 2 weeks after surgery. Water maze training was followed within $2-3 \mathrm{~d}$ by passive avoidance training.

Surgery and histology. Surgical procedures were similar to those described for experiments 1-3 except that different lesion coordinates were used. For PH lesions $(n=10), 10$ injections of $25-50 \mathrm{nl}$ of ibotenic acid were made at each of the following stereotaxic coordinates: $2.3,3.3,4.3$, $5.2,5.2,6.4,6.4,7.1,7.1$, and $8.9 \mathrm{~mm}$ posterior to bregma; $6.3,6.4,6.5$, $6.6,6.6,6.7,6.7,6.1,6.1$, and $6.0 \mathrm{~mm}$ lateral from the midline; and 8.0, $8.0,8.0,8.0,7.0,7.1,6.1,6.2,5.2$, and $6.0 \mathrm{~mm}$ below the skull surface, respectively. For HC lesions $(n=8), 12$ injections of $100 \mathrm{nl}$ ibotenic acid were made bilaterally at each of the following stereotaxic coordinates: $2.2,3.0,3.0,3.0,4.0,4.0,4.0,5.0,5.0,5.8,5.8$, and $5.8 \mathrm{~mm}$ posterior to bregma; 1.0, 1.4, 1.4, 3.0, 2.6, 2.6, 3.8, 4.6, 4.6, 4.1, 5.0, and 5.0 mm lateral from the midline; and 3.8, 3.7, 3.0, 3.4, 3.8, 2.9, 3.6, 7.0, 4.0, 4.4, 6.4, and $4.4 \mathrm{~mm}$ below the skull surface, respectively. At the end of behavioral testing, subjects were deeply anesthetized and perfused. Tissue was processed as described previously with the exception that coronal sections at $480 \mu \mathrm{m}$ intervals for both $\mathrm{PH}$ and $\mathrm{HC}$ lesions were used to assess the amount of tissue damage.

Behavioral apparatus and procedures. The apparatus and behavioral procedures for the Morris water maze were the same as described for experiment 1 . The passive avoidance apparatus (Stoelting Co., Wood Dale, IL) consisted of a white Plexiglas chamber connected to a black Plexiglas chamber via a sliding guillotine door. The floor consisted of a grid interfaced with a current scrambling controller for foot shock administration. Passive avoidance consisted of an acquisition trial on the first (training) day and a retention trial $24 \mathrm{hr}$ later. On the training day, animals were placed in the lighted chamber. After $10 \mathrm{sec}$, the door was opened, and latency to enter the dark chamber was recorded. An animal was considered to have entered the dark chamber when all fours paws were beyond the guillotine door. After entry, the door was closed. After 2 sec had elapsed, a shock stimulus was delivered. The shock level and duration were set at $1 \mathrm{~mA}$ for $2 \mathrm{sec}$. The rat was immediately removed back to its home cage. Twenty-four hours later, a retention test consisted of placing the rat back into the lighted chamber, waiting $10 \mathrm{sec}$, opening the door, and timing latency of the rat to enter the dark chamber. If a rat did not enter the chamber in $540 \mathrm{sec}$, the trial was terminated, and the rat was removed to the home cage.

Data analysis. Performance on the Morris water maze was analyzed as described for experiment 1 to make comparisons across experiments. Additionally, for probe trials we analyzed platform crossings and time in training quadrant because these measures have traditionally been used to assess deficits on the water maze associated with hippocampal damage. For passive avoidance, latency to enter the dark chamber on the retention day was analyzed using rANOVA with group as a between-subjects variable. For rANOVA, planned comparisons of significant main effects and interactions were assessed with appropriate contrasts.

\section{Results}

\section{Experiment 1: effects of PER and POR lesions on place} learning in the water maze

In this experiment, rats with bilateral neurotoxic lesions of perirhinal or postrhinal cortex that were impaired on contextual fear conditioning (Bucci et al., 2000) were tested in a standard place training task in the Morris water maze. The goal was to examine the contribution of the perirhinal and postrhinal cortices to spatial learning in lesioned animals that were known to be impaired on a measure of contextual learning.

\section{Histology}

In the PER group, damage to the PER was present on $85.3 \%$ of the sections analyzed. There was some evidence of CA1 damage in the PER subjects as reported previously (Bucci et al., 2000). Damage to the POR was bilateral and extended throughout the rostrocaudal extent of the POR and was observable on $77.4 \%$ of the sections analyzed. The largest and smallest lesions were as reported previously (Bucci et al., 2000).

\section{Behavior}

Subjects in this experiment were used in a previous behavioral study. In that study, both prerhinal and postrhinal lesions of either perirhinal or postrhinal cortex produced significant impairment in contextual fear conditioning (Bucci et al., 2000). In the present study, damage to either the PER or POR had little or no effect on acquisition of place learning in the Morris water maze (Fig. 1). Analyses of latency to reach the training platform indicated that there was no main effect of group $(p>0.41)$ and no group by block interaction $(p>0.41)$. Likewise, there was no main effect of group $(p>0.40)$ and no group by block interaction $(p>0.68)$ for cumulative distance (data not shown).

There were some effects of lesions on learning measures as assessed in probe trials when the hidden platform was removed (Fig. $1 B$ ). A group difference was observed in average proximity to the platform location $\left(F_{(2,34)}=4.3 ; p<0.02\right)$, and there was a trend toward a group by block interaction $(p>0.09)$. Post hoc analysis indicated that the PER group had a significantly larger average proximity to the platform location as compared with the 
CTL group $(p<0.01)$. There was also a group by block interaction for the PER versus CTL comparison $(p<0.02)$, with PER rats showing greater deficits later in training. Post hoc analysis indicated that the PER versus CTL difference was restricted to the third probe trial $(p<0.001)$, with a nonsignificant trend toward a difference on the fourth probe trial $(p>0.06)$. The POR group was not different from the CTL group ( $p>0.89)$, nor was there a group by block interaction $(p>0.31)$.

The PER impairment was confirmed by an analysis of the spatial learning index. There was an overall effect of group $\left(F_{(2,34)}=4.77 ; p<0.02\right)$. PER rats had significantly higher (worse) indexes as compared with the CTL rats $(p<0.01)$. POR rats were not significantly different from CTL rats $(p>0.91)$.

The results of cue learning were surprising. The PER group had the shortest latencies to reach the visible platform and the shortest cumulative distance (supplemental Fig. $1 \mathrm{~A}$, available at www.jneurosci.org). A main effect of group on latency $\left(F_{(2,34)}=\right.$ $5.43 ; p<0.009)$ was followed by post hoc analysis, indicating that the PER group had significantly shorter latencies than the POR group $(p>0.002)$ and marginally significantly shorter latencies than the CTL group $(p<0.052)$. A main effect of group on cumulative distance $\left(F_{(2,34)}=5.17 ; p<0.01\right)$ was followed by post hoc analysis indicating that the PER group exhibited a significantly shorter cumulative distance to the visible platform than the POR group ( $p>0.003)$ and a marginally significantly shorter cumulative distance as compared with the CTL group $(p>0.07)$. Analyses of swim speed indicated no group differences during place learning training trials $(p>0.39)$.

Correlation analyses were conducted to examine the relationship between place learning in the water maze and contextual learning in the same animals from a previous study. High percentages of freezing represent good retention of the association between context and the shock stimulus acquired in the training session. Correlation analyses between the spatial learning index (present study) and the mean of the first four blocks of extinction (Bucci et al., 2000) indicated that spatial and contextual learning were significantly negatively correlated $(r=-0.42 ; p<0.02)$ when the analysis included rats from all three groups (Fig. 1C). Rats with higher (worse) spatial learning indexes showed poorer contextual learning. Correlations within each of the CTL, PER, and POR groups, however, were not significant $(r=0.18,-0.22$, and -0.28 , respectively).

\section{Experiment 2: effects of PER and POR lesions on place and incidental learning in the water maze}

In experiment 1 , rats with perirhinal damage exhibited a mild deficit on standard place training in the Morris water maze, but rats with postrhinal damage did not. Because of variability across studies and laboratories, we sought to replicate these findings using the standard place training task and extend them to another procedure conducted in the water maze: incidental learning. Because the hippocampus may be involved in incidental learning (Good et al., 1998), we hypothesized that the cortical regions projecting to the hippocampus might be involved in processing information about spatial cues in a task in which those cues were not relevant to normal performance. Rats were trained on a visible platform and then examined for the incidental acquisition of knowledge about the spatial location of a visible platform on the basis of spatial cues (Rapp et al., 1987). Rats used in the present study were shown in a previous study to have deficits in contextual discrimination (Bucci et al., 2002).
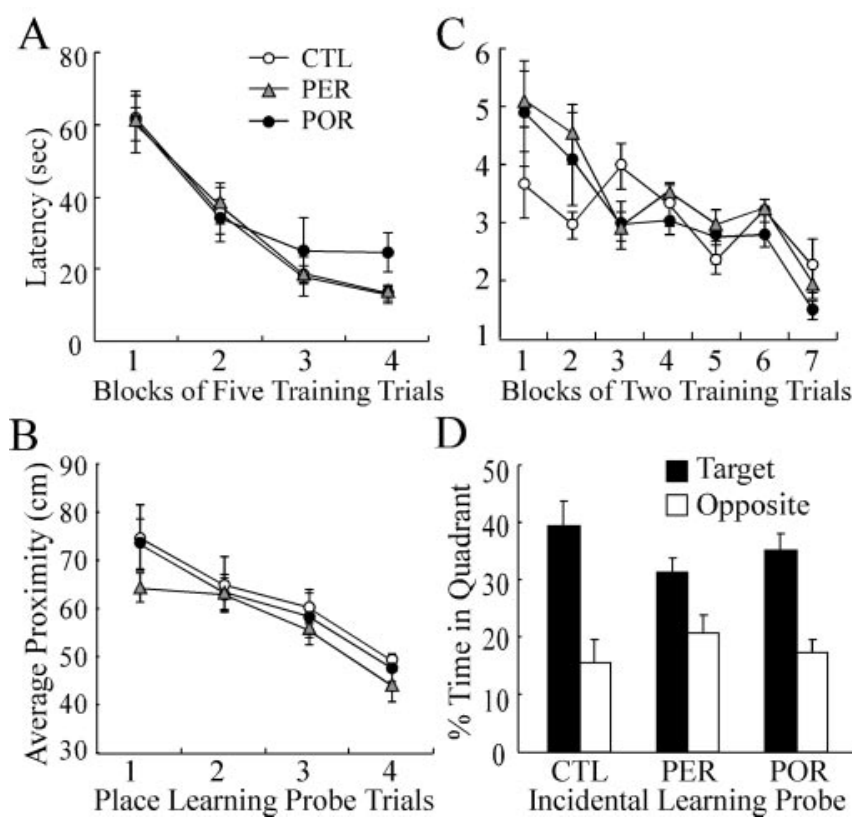

$\mathrm{D}$

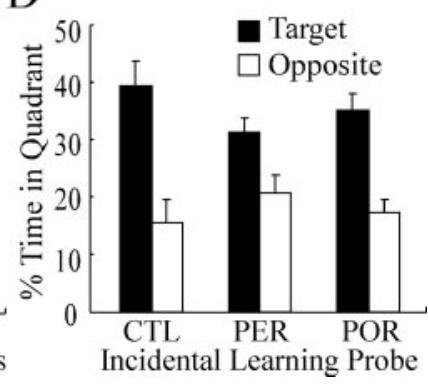

Figure 2. Effects of damage to PER or POR on two water maze tasks in experiment 2. Place learning is shown in $A$ and $B$, and incidental learning is shown in C and $D$. $A$, Latency to find the hidden platform during training trials. $B$, Average proximity to the platform during probe trials. C, Latency to swim to the visible platform during training trials. D, Time spent in each quadrant of the water maze during the final (probe) trial during incidental learning in the water maze. There were no group differences on either task. CTL, Control group; PER, perirhinal-lesioned group; POR, postrhinal-lesioned group. Data are mean \pm SEM.

\section{Histological results}

Two rats were excluded from the study because POR damage was unilateral. In the remaining eight rats, damage to POR was bilateral and extensive ( $80 \pm 5 \%$ of the sections analyzed). In the PER-lesion group, one rat was excluded because of bilateral sparing of perirhinal cortex, leaving nine rats included in the study. Four rats sustained substantial and extensive bilateral damage to perirhinal cortex but were excluded from quantitative lesion analysis because of missing sections. In the five remaining rats, damage to PER was extensive ( $54 \pm 7 \%$ of the sections analyzed). Only minor, unilateral damage was observed outside the target region. No evidence of hippocampal damage was observed for PER or POR rats. Three sham-control rats were excluded from the behavioral analyses because the brains exhibited significant mechanical damage to the neocortex, leaving six animals in the sham group.

\section{Cue learning}

One rat in the PER group was $>2.5$ SDs above the group mean on latency to reach the visible platform and on cumulative distance to reach the platform. This rat was excluded from all further analyses. All remaining rats learned to swim directly to the visible platform during cue training (supplemental Fig. $1 B$, available at www.jneurosci.org). Perirhinal or postrhinal damage had no effect on performance as indicated by the lack of significant group differences in latency $(p>0.52)$ or cumulative distance $(p>$ $0.89)$ to reach the visible platform. There was a marginally significant tendency toward a group difference in swim speed during cue training $(p<0.11)$ and training trials $(p<0.08)$ but not during probe trials $(p>0.60)$.

\section{Place training}

During place training, rats in all three groups rapidly learned the location of the escape platform. For all groups, latency to locate 

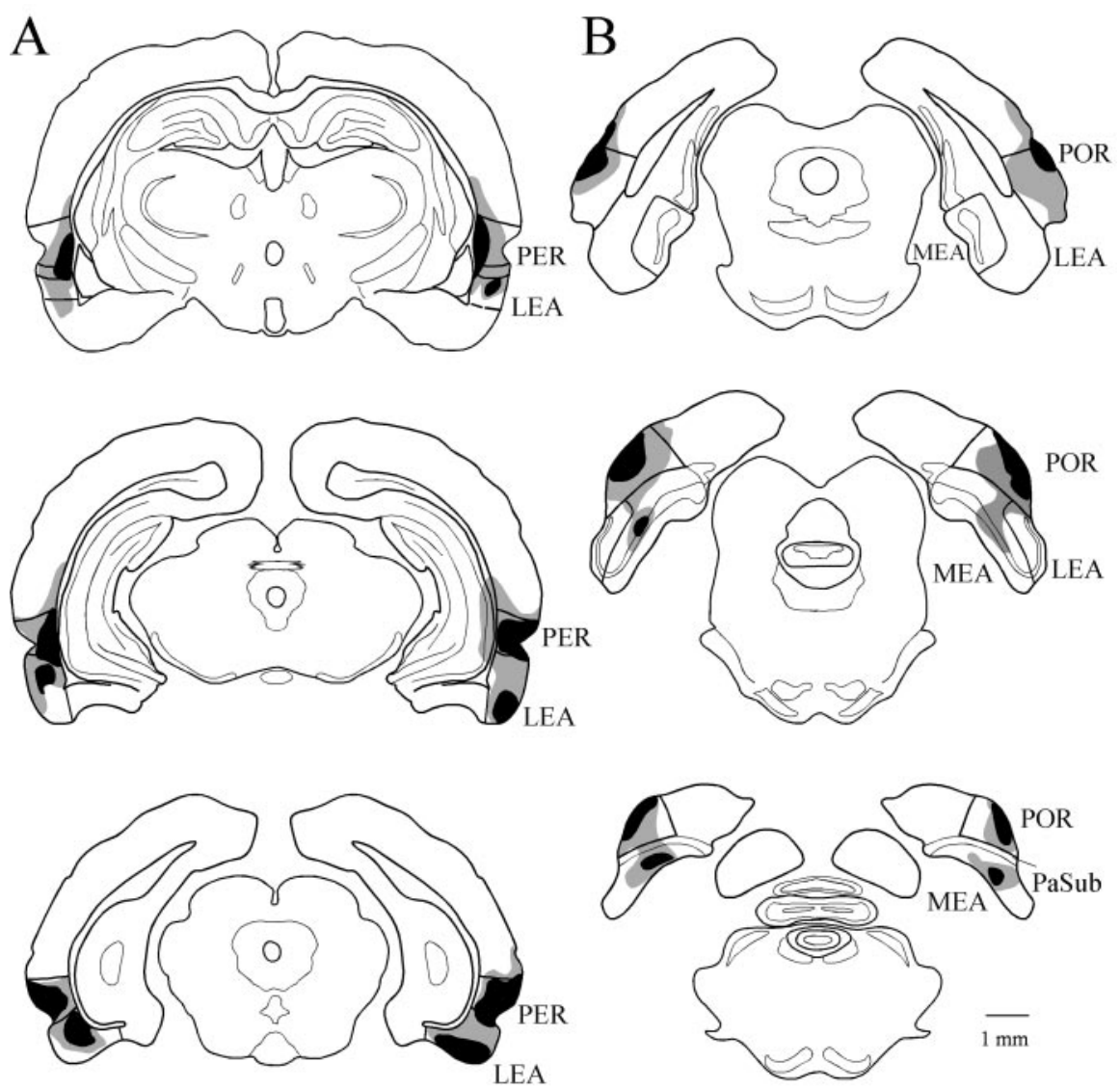

Figure 3. PER/LEA and POR/MEA lesions. A, Schematic of the largest (gray) and smallest (black) PER/LEA neurotoxic lesions. $B$, Schematic of the largest (gray) and smallest (black) POR/MEA neurotoxic lesions. LEA, Lateral entorhinal cortex; MEA, medial entorhinal cortex; PaSub, parasubiculum; PER, perirhinal cortex; POR, postrhinal cortex.

the hidden platform decreased over the course of training (Fig. $2 A)$. There was no main effect of group $(p>0.81)$ and no group by block interaction $(p>0.93)$. Performance during the probe trials was also comparable across groups (Fig. $2 B$ ). There was no significant effect of group ( $p>0.26)$ and no group by trial interaction $(p>0.93)$ in average proximity to the platform location during probe trials.

Again, correlation analyses were conducted to examine the relationship between place learning in the water maze and contextual learning in the same animals from a previous study. The spatial learning index and the difference in percentage of freezing between the shock and no shock conditions were not significantly correlated for all animals $(r=0.02 ; p>0.73)$. Additionally, correlations within each of the CTL, PER, and POR groups were not significant $(r=-0.36,-0.40$, and 0.41 , respectively; $p>$ $0.30)$.

\section{Incidental learning}

The performance of control and lesioned rats was comparable during the cue-training trials in which the escape platform was visible. The latency to reach the visible platform decreased over training days for rats in all groups (Fig. 2C), but there was a marginally significant group by day interaction $\left(F_{(12,114)}=1.83\right.$; $p<0.052)$ without a main effect of group $(p>0.54)$. Post hoc contrasts at each day revealed no significant differences between groups; however, the PER and POR groups exhibited marginally shorter latencies as compared with the CTL group on day $3(p<$ 0.07). Additionally, the POR group exhibited a marginally shorter latency than the PER group to reach the visible platform on day 6 ( $p<$ $0.07)$.

During the probe trial on day 7 , both control and lesioned rats exhibited a spatial bias, as illustrated in Figure 2D. The percentage of time spent in the training quadrant was significantly greater than chance for rats in all groups (controls: $t_{(8)}$ $=-3.50, p<0.02$; PER-lesioned: $\mathrm{t}_{(7)}=$ -2.69, $p<0.03$; POR-lesioned: $t_{(8)}=$ $-3.46, p<0.01)$. Additionally, there was no difference between groups in proximity to the target location $(p>0.14)$, the percentage of path length in the training quadrant $(p>0.22)$, or the percentage of time spent in the training quadrant $(p>$ $0.27)$.

\section{Experiment 3: effects of PER/LEA and POR/MEA lesions on place learning in the water maze and contextual discrimination}

In experiments 1 and 2, POR rats were unimpaired and PER rats were mildly impaired or unimpaired on place learning in the water maze. One possibility is that the redundancy in the parallel corticohippocampal pathways permits sufficient sensory and spatial information to reach the hippocampus despite PER or POR damage. Because the perirhinal-lateral entorhinal processing stream carries more information about individual stimuli to the hippocampus whereas the postrhinal-medial entorhinal stream carries more spatial information, damage to the medial processing stream might disrupt performance on a spatial task more effectively than damage to the lateral processing stream. Thus, in experiment 3 we examined the effects of PER/LEA or POR/MEA lesions on place learning in the water maze and on contextual fear discrimination.

\section{Histology}

One PER/LEA and two POR/MEA rats died in surgery. One PER/ LEA subject was excluded because cortical damage was unilateral, leaving a total of five in the group. In the remaining subjects, damage to PER/LEA was bilateral and extended throughout the rostrocaudal extent of PER/LEA (75 $\pm 8 \%$ of the sections analyzed), as illustrated by the schematic in Figure $3 A$. Additionally, minor damage at the border of the adjacent temporal association cortex $\left(\mathrm{Te}_{\mathrm{v}}\right)$ was noted on $60 \pm 16 \%$ of the sections. One POR/ MEA subject was excluded because cortical damage was restricted to one hemisphere. In the remaining six POR/MEA subjects, damage to POR/MEA was bilateral and extended throughout the rostrocaudal extent of the region. Damage was apparent on $89 \pm$ $6 \%$ of the sections analyzed, as illustrated in Figure $3 B$. The lesion encroached slightly and unilaterally into the adjacent $\mathrm{Te}_{\mathrm{v}}$ on $60 \pm$ $11 \%$ of the sections.

\section{Behavior}

Both naive and sham groups learned the discrimination as indicated by a significant main effect of shock condition on the test day $\left(F_{(1,14)}=6.52 ; p<0.023\right)$. Discrimination between contexts 
was not significantly different between the two control groups at any point during training $(p>0.24)$ or testing $(p>0.31)$. Data from the two groups was thus combined (CTL) for all further analyses.

Lesioned rats were not significantly different from control rats on the two training days as indicated by nonsignificant effects of group $(p>0.23)$ and nonsignificant interaction of group with shock condition $(p>0.62)$. On the test day, there were important differences in the amount of freezing exhibited by control and lesioned rats, as indicated by a significant interaction between group and shock condition $\left(F_{(2,26)}=3.85 ; p<\right.$ $0.034)$, but no overall effect of group $(p>$ 0.15 ) (Fig. 4A). Rats in the control group froze more in the shock context than in the no-shock context. The PER/LEA and POR/MEA groups showed no evidence of learning the discrimination. Post hoc analyses of freezing in the two contexts on the test day were conducted individually for each group to determine which groups were discriminating significantly between contexts. These analyses revealed an effect of context for the control group $(p>0.02)$ but not the PER/LEA-lesioned group $(p>0.61)$ or the POR/MEA-lesioned group $(p>0.62)$.

In contrast, on place learning in the Morris water maze, damage to either the PER/LEA or POR/MEA had no effect on acquisition, as assessed by training trials or probe trials (Figs. $4 A, B$ ). There was no main effect of group on training trial latency to reach the training platform $(p>0.63)$ or on cumulative distance ( $p>0.63$; data not shown), nor was there a group by block interaction for either latency $(p>0.50)$ or cumulative distance $(p>0.45)$. During probe trials, there was no main effect of group on proximity to the platform location $(p>0.67)$ and no group by block interaction $(p>0.68)$. The lack of impairment in the lesion groups was confirmed by an analysis of the learning index, i.e., there was no main effect of group ( $p>0.79)$.

The PER/LEA group had, numerically, the shortest latencies to reach the visible platform and the shortest cumulative distance (supplemental Fig. 1C, available at www.jneurosci.org); however, there was no main effect of group for latency $(p>0.18)$ or cumulative distance $(p>0.17)$. Because rats with perirhinal lesions were significantly facilitated on cue learning in experiment 1 , post hoc analyses were conducted. The PER/LEA group was marginally different from the CTL group on cue latency $(p<0$. $07)$ and cumulative distance to the visible platform $(p<0.06)$.

Analyses of swim speed indicated a main effect of group for training trial swim speed $\left(F_{(2,20)}=3.83 ; p<0.03\right)$ and for probe trial swim speed $\left(F_{(2,20)}=4.14 ; p<0.03\right)$. Post hoc analyses indicated that the POR/MEA group was significantly slower than the PER/LEA and CTL groups for training trials $(p<0.02)$ and probe trials $(p<0.05)$.

A final analysis was conducted to determine whether there was a correlation between performance on the water maze as indicated by the spatial learning index and performance on the contextual discrimination task. Correlation analysis indicated that the spatial learning index and the difference in percentage of freezing between the shock and no-shock conditions was not significantly correlated for all animals $(r=-0.10 ; p>0.65)$. Correlations within each of the CTL, PER/LEA, and POR/MEA groups were also not significant $(r=-0.01,0.01$, and -0.46 , respectively; $p>0.25$ ).

\section{Experiment 4: effects of combined PER/POR/EC lesions on place learning on the water maze and passive avoidance learning}

Experiment 3 showed that damage to either the POR/MEA or the PER/LEA pathway was sufficient to damage performance on contextual fear discrimination but not on spatial learning in the Morris water maze. One possibility is that sensory input via either pathway is sufficient to support spatial learning but that a lesion of both pathways would result in deficits. Indeed, both the postrhinal and perirhinal cortices receive input from visual association cortices (Burwell and Amaral, 1998b; Burwell, 2001). Thus, in this experiment, rats received combined lesions of the perirhinal, postrhinal, and entorhinal cortices $(\mathrm{PH})$ and were tested on spatial and contextual learning. An HC lesion group was included for comparison. If spatial and contextual learning are similarly supported by corticohippocampal circuitry, both the large parahippocampal lesions and the hippocampal lesions should result in deficits in both tasks. Moreover, indexes of performance on the two tasks should be strongly correlated. The spatial task was the same water maze task as that used in experiments 1-3. As a measure of contextual learning, passive avoidance was used (StubleyWeatherly et al., 1996). Although passive avoidance has an instrumental component, it is similar to the other contextual learning tasks in that it requires the association between a context and an aversive stimulus.

\section{Histology}

Two PH subjects were excluded because of limited damage to the target regions, leaving a total of eight subjects in the group. In the remaining subjects, damage to the perirhinal, postrhinal, and entorhinal cortices was bilateral and extended throughout the rostrocaudal extent of the regions (Fig. 5A). There was some sparing of dorsal perirhinal cortex at rostral levels. Damage outside the target regions was limited. Three HC lesioned subjects were eliminated because there was substantial unilateral sparing of the hippocampus. In all remaining subjects, damage was extensive and bilateral (Fig. 5B).

\section{Behavior}

In the Morris water maze, the combined perirhinal, postrhinal, and entorhinal damage in the $\mathrm{PH}$ group had no effect on acquisition of place learning as assessed by training trials or probe trials 

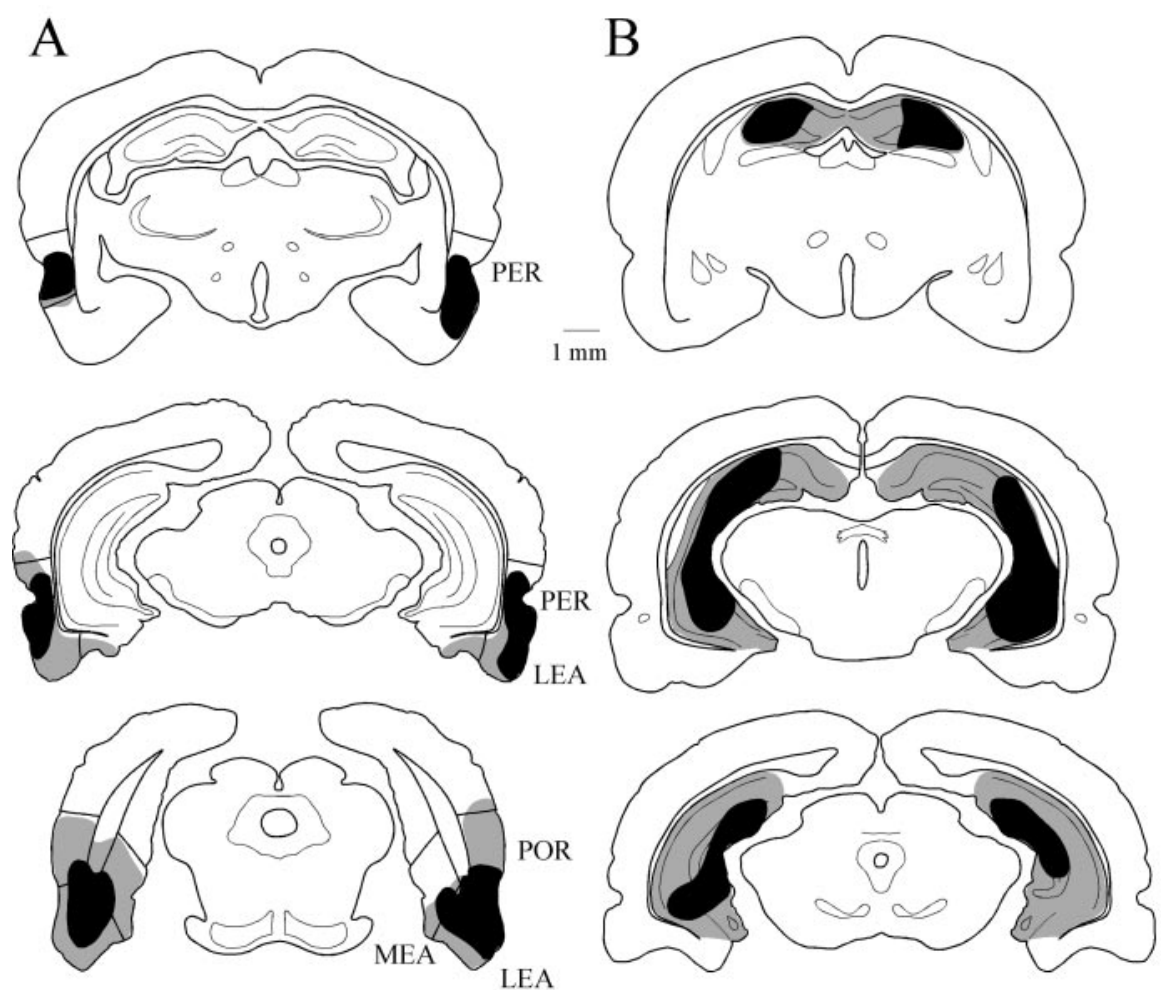

Figure 5. Parahippocampal and hippocampal lesions. $A$, Schematic of the largest (gray) and smallest (black) parahippocampal neurotoxic lesions. $B$, Schematic of the largest (gray) and smallest (black) hippocampal neurotoxic lesions. LEA, Lateral entorhinal cortex; MEA, medial entorhinal cortex; PER, perirhinal cortex; POR, postrhinal cortex.

(Figs. 6A, $B$ ). In contrast, hippocampal damage was associated with impaired place learning, which was clearly evident in performance during probe trials. There was no main effect of group on training trial latency to reach the training platform $(p>0.34)$ or on cumulative distance ( $p>0.28$; data not shown), nor was there a group by block interaction for either latency $(p>0.61)$ or cumulative distance $(p>0.60)$. During probe trials, however, there was a main effect of group on proximity to the platform location $\left(F_{(2,19)}=4.12\right.$; $\left.p<0.033\right)$ but no group by block interaction $(p>0.59)$. Despite the small number of HC subjects, contrasts indicated that the HC group was significantly impaired relative to the CTL group on this measure $(p<0.011)$, but the $\mathrm{PH}$ group was not $(p>0.13)$. The HC impairment was confirmed by an analysis of the learning index. There was an overall effect of group $\left(F_{(2,19)}=4.03 ; p<0.035\right)$. HC rats had significantly higher (worse) spatial learning indexes as compared with the CTL rats $(p<0.01)$. PH rats were not significantly different from CTL rats $(p>0.20)$.

Because the impairment in the HC group was less severe than expected, two additional measures of probe trial performance were assessed to further characterize place learning. These included two traditional water maze measures: time in the training quadrant and number of platform location crossings. There was a significant main effect of group on time spent in the training quadrant during the four probe trials $\left(F_{(2,19)}=4.92 ; p<0.02\right)$. Planned contrasts indicated that the $\mathrm{HC}$ group was significantly impaired relative to the CTL group on this measure $(p<0.0009)$, but the PH group was not $(p>0.09)$. Likewise, there was a significant main effect of group on number of times the animals crossed the platform location during the four probe trials $\left(F_{(2,19)}\right.$ $=6.00 ; p<0.009)$. Planned contrasts indicated that the HC group was significantly impaired relative to the CTL group on this measure $(p<$ $0.003)$, but the $\mathrm{PH}$ group was not $(p>$ $0.16)$.

There were no main effects of group on measures of performance on the cue learning task (supplemental Fig. $1 \mathrm{D}$, available at www.jneurosci.org). The $\mathrm{PH}$-lesioned group exhibited, numerically, the shortest latencies to reach the visible platform and the shortest cumulative distance, but there were no main effects of group for latency $(p>0.42)$ or cumulative distance $(p>$ $0.68)$. Because rats with perirhinal lesions were significantly facilitated on cue learning in experiment 1, post hoc analyses were conducted. The PH group was not different from the CTL group on cue latency $(p>0.19)$ or cumulative distance to the visible platform $(p>0.41)$. An additional analysis indicated that there were no group differences in swim speed during training trials $(p>0.92)$.

For passive avoidance, mean retention scores for the $\mathrm{PH}$ and the $\mathrm{HC}$ groups were numerically less than those for the CTL group (Fig. 6C). ANOVA indicated a significant effect of group on retention latency $\left(F_{(2,19)}=3.54 ; p<0.05\right)$. Post hoc analyses, however, indicated that the CTL group was significantly different from the $\mathrm{PH}$ group $(p<0.02)$ but not the $\mathrm{HC}$ group $(p>0.17)$. The $\mathrm{PH}$ and $\mathrm{HC}$ groups were not different from each other $(p>0.40)$. The retention scores of $\mathrm{HC}$ subjects were numerically lower than those of CTL subjects and similar to those of the PH subjects; however, the lack of a statistically significant difference between CTL and HC groups was surprising given previous reports that passive avoidance is sensitive to hippocampal damage (Stubley-Weatherly et al., 1996). In the present study, the lack of statistical difference was likely attributable to a lack of statistical power. Three subjects with hippocampal lesions were deleted because of partial sparing in one hemisphere, leaving only five subjects in the HC group. To address the issue of whether the lack of significance was caused by a lack of power, we reanalyzed the data including the HC subjects with partial unilateral sparing of the hippocampus. The results revealed that the $\mathrm{HC}$ group was significantly different from the CTL group $(p<0.03)$ but not the PH group $(p>0.27)$, a result that is consistent with previous studies.

A final analysis was conducted to determine whether there was a correlation between performance on the water maze as indicated by the spatial learning index and performance on the passive avoidance test (Fig. 6D). Passive avoidance was not significantly related to spatial learning by these measures $(r=0.003$, $p>0.98)$. Post hoc analyses of within-group correlations also did not reveal a significant relationship $(p>0.30)$.

\section{Discussion}

Several theories of the neural basis of memory have addressed the functional relationships among the hippocampus and the cortical regions that surround it (Eichenbaum et al., 1994; ZolaMorgan et al., 1994; Rudy and Sutherland, 1995; Aggleton and Brown, 1999; Aggleton et al., 2000), but a clear understanding has 

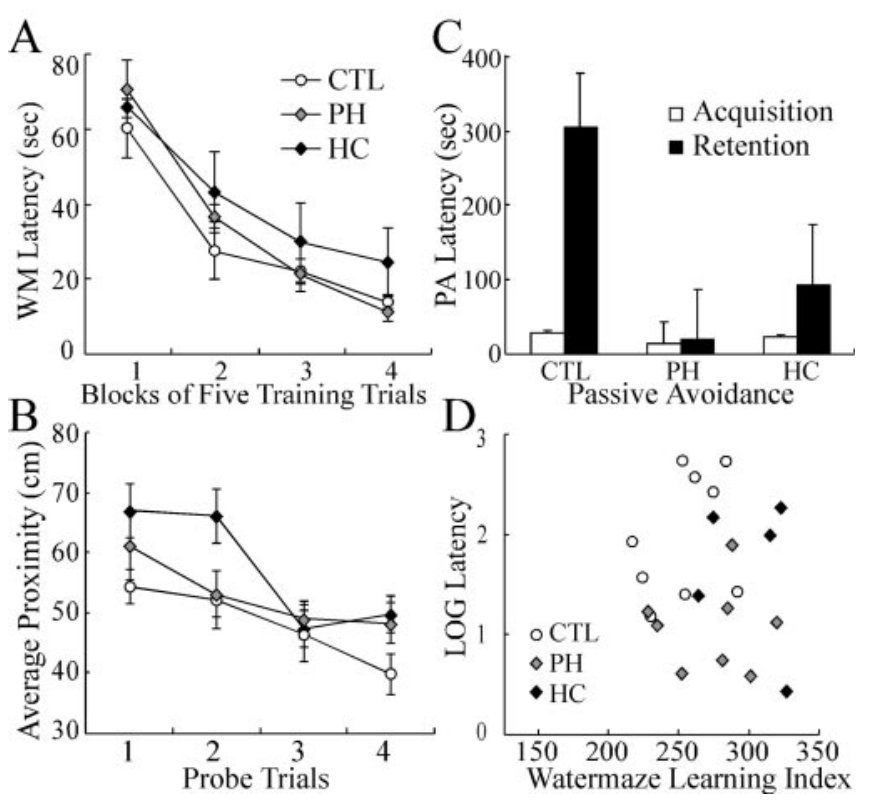

Figure 6. Effects of damage to $\mathrm{PH}$ - or HC-lesioned rats on the Morris water maze task and on passive avoidance in experiment $4 . A$, Latency to find the hidden platform during training trials. $B$, Average proximity to the platform during probe trials. Similar to findings for the smaller lesions, latency to find the platform decreased during training but did not differ between the PH and CTL groups. Likewise, proximity to the platform location during probe trial performance was comparable for the PH and CTL groups. In contrast, the HC-lesioned rats were significantly impaired during training and on probe trials. C, Latency to enter the dark chamber on retention day of passive avoidance testing for $\mathrm{CTL}, \mathrm{PH}$, and $\mathrm{HC}$ lesion groups. Data are mean \pm SEM. $D$, Relationship between log latency to enter the dark chamber in passive avoidance and performance on the water maze as indicated by a spatial learning index (see Results for details). Performance on the two tasks was not significantly correlated. CTL, Control group; HC, hippocampal-lesioned group; $\mathrm{PH}$, combined perirhinal, postrhinal, lateral entorhinal, and medial entorhinal-lesioned group.

not emerged. We examined this issue by assessing animals with various lesions of corticohippocampal circuitry on tests of spatial and contextual learning. Rats with component lesions of the parahippocampal region were essentially unimpaired on place learning in the Morris water maze but consistently showed deficits on three different contextual learning tasks, including contextual fear conditioning, contextual discrimination, and passive avoidance.

Experiments by LeDoux and colleagues (Corodimas and LeDoux, 1995; Phillips and LeDoux, 1995) provided evidence that post-training perirhinal lesions impair expression of conditioned freezing, although pretraining lesions were reported not to affect contextual fear conditioning. Several previous studies using reversible inactivation procedures support a role for the perirhinal cortex in the consolidation of contextual fear (Sacchetti et al., 1999, 2002; Tassoni et al., 1999). In contrast, two studies by Herzog and Otto $(1997,1998)$ suggested that pretraining lesions of the perirhinal cortex do not affect contextual conditioning. Those studies, however, lesioned only anterior perirhinal cortex. In our hands, lesions must involve the full rostrocaudal extent of the perirhinal cortex to be behaviorally effective. Previous studies using entorhinal lesions have shown both deficits in contextual fear conditioning (Maren and Fanselow, 1997) and spared acquisition of contextual freezing (Phillips and LeDoux, 1995). Taken together, the weight of the evidence supports a role in contextual learning for the cortical regions that surround the hippocampus.

Previous studies of parahippocampal contributions to performance on the Morris water maze have yielded mixed results. One study using excitotoxic lesions of the postrhinal cortex reported mild deficits in the water maze (Liu and Bilkey, 2002), but a study using excitotoxic perirhinal plus postrhinal lesions showed no impairment (Bussey et al., 1999). Perirhinal electrolytic lesions resulted in mild deficits (Wiig and Bilkey, 1994a,b; Liu and Bilkey, 1998). When excitotoxic perirhinal lesions were used, deficits were even less pronounced (Liu and Bilkey, 2002) or performance was spared (Bussey et al., 1999; Mumby and Glenn, 2000). Assessment of spatial learning using other paradigms in the water maze, for example, delayed-matching-to-place tasks, have also shown spared performance (Glenn and Mumby, 1998) or impaired performance (Liu and Bilkey, 2002). Several previous studies found that electrolytic lesions of the entorhinal cortex (Schenk and Morris, 1985; Nagahara et al., 1995; Liu and Bilkey, 1998; Glasier et al., 1999) or disconnection of the entorhinal cortex and dentate gyrus (Skelton and McNamara, 1992) produced impairments in the water maze (Hagan et al., 1992). Other studies using neurotoxic methods to lesion the entorhinal cortex showed a deficit, but in those studies damage extended into the subiculum (Good and Honey, 1997; Oswald and Good, 2000).

Here, we showed a consistent dissociation between the effects of parahippocampal damage on spatial and contextual learning. Subjects with lesions including damage to postrhinal cortex (experiments 1-4), perirhinal cortex (experiments 2-4), and entorhinal cortex (experiments 3 and 4) performed normally on place learning in the water maze but were impaired on tests of contextual learning. In experiment 1 , rats with perirhinal damage were mildly impaired in place learning; however, hippocampal damage was observed for some subjects in the perirhinal lesion group.

Lesion methods likely contribute to the variability in effects of parahippocampal lesions across studies and laboratories. Although neurotoxic lesion methods are less likely to yield deficits because of damage to fibers of passage, they have been associated with a lack of regional specificity (Jarrard, 2002) and thus have the disadvantage of possibly producing hidden damage in the hippocampus. This is more likely to be an issue in perirhinal lesion studies because the perirhinal cortex lies close to the midseptotemporal and temporal levels of the hippocampus. In the present study, we found a deficit with perirhinal lesions in experiment 1 but not experiment 2. Notably, the perirhinal lesions were larger, and there was evidence of CA1 damage in experiment 1. Thus, it may be that in some cases parahippocampal lesions lead to deficits in place learning caused by unintended hippocampal damage.

Other procedural differences may also contribute to the variable results across studies. Behavioral paradigms can vary in the extent to which they require highly processed sensory associational input. For the water maze, if distal visual cues are simple, as in the present experiments in which simple cues were placed on curtains surrounding the water maze, information processing by intact cortical polymodal associational regions may not provide an advantage for control subjects, and subcortical sensory input may adequately support performance. When the water maze is surrounded by more complicated spatial cues, for example, features of an open room, the increased complexity of the cues may recruit higher-order cortical polymodal association regions. This is consistent with the positive findings for postrhinal cortex (Liu and Bilkey, 2002) and perirhinal cortex (Liu and Bilkey, 1998) in two studies in which the maze was placed in an open room with laboratory furniture, lights, and other prominent visual cues. It is also possible that the varied effects of parahippocampal damage on spatial versus contextual learning reflects differences in task difficulty. Despite procedural differences, however, it is still the 
case that hippocampal damage reliably affects spatial navigation, whereas parahippocampal damage more reliably affects contextual learning. Thus, in the present study, in which animals were tested for both types of learning, a dissociation in spatial and contextual learning was evident.

Previous theories of hippocampal function proposed that contextual and spatial learning share a common underlying process (O'Keefe and Nadel, 1978; Nadel and Willner, 1980). Another proposal is that hippocampal deficits in these two types of learning may have different origins (Good and Honey, 1997). Here, we provide evidence that contextual and spatial learning have different neural substrates and that the parahippocampal regions contribute more to the former than the latter. Alternatively, parahippocampal regions may contribute similarly to spatial and contextual learning, despite the lack of a consistent significant impairment in place learning after parahippocampal lesions. In that case, we would expect learning in the two types of tests to be correlated. In the present study there was a significant correlation between spatial and contextual learning in only one of four experiments (experiment 1 in which hippocampal damage was observed), and in that experiment, within-group correlations were not significant. Because there was some amount of hippocampal damage in that experiment, and because there was no correlation between tasks in experiment 2-4, the balance of the evidence is against the hypothesis that parahippocampal regions contribute similarly to spatial and contextual learning.

We propose that spatial and contextual learning are supported, at least in part, by different neural substrates. Specifically, contextual learning requires the hippocampus and the cortical regions that surround it, but spatial functions can operate independently of highly processed neocortical sensory input. This notion is similar to ideas that have been proposed previously (Aggleton and Brown, 1999; Aggleton et al., 2000). It may be that certain tasks can be solved by a spatial navigation system that requires some spatial input but does not require processing by higher-order sensory associational regions. In other words, there may be a component process sufficient for successful performance on the Morris water maze that is not sensitive to damage of the parahippocampal region; subcortical input to the hippocampus may be sufficient for spatial navigation, especially when spatial cues are simple.

This notion is consistent with the proposal that the hippocampus may be part of a specialized spatial navigation system (Muller et al., 1999). In some situations, vestibular and self-motion cues can provide the primary drive on place cells and head direction cells, overriding visual landmark cues (Knierim et al., 1998). The circuitry of regions linking areas in which neuronal correlates of spatial behavior have been identified could support sufficient sensory input to the hippocampus without parahippocampal associational input. Specifically, areas in which head direction cells have been identified, such as the anterior dorsal thalamus, lateral dorsal thalamus, and the lateral mammillary nucleus, all provide direct input to the dentate gyrus (Amaral and Witter, 1995; Taube, 1998). Spatial navigation under some conditions could be accomplished with rudimentary processing of visual landmarks provided by the sensory input to the thalamus and hypothalamus. Thus, sensory input to the hippocampus via the fornix is sufficient to support the sensory processing needs for navigation in a water maze task. In contrast, some contextual learning tasks may require more detailed processing of stimulus features. In those cases, subcortical input is not sufficient for normal performance on the task.

These findings indicate that the cortical regions surrounding the hippocampus contribute differentially to spatial and contextual learning, suggesting that these functions are supported by different neuroanatomical substrates. Although spatial navigation is a reliable assay of hippocampal function, place learning in the Morris water maze is not reliably sensitive to parahippocampal damage. Thus, contextual learning tasks provide a better test of memory when the regions of interest include the cortical regions that surround the hippocampus.

\section{References}

Aggleton JP, Brown MW (1999) Episodic memory, amnesia, and the hippocampal-anterior thalamic axis. Behav Brain Sci 22:425-489.

Aggleton JP, Vann SD, Oswald CJ, Good M (2000) Identifying cortical inputs to the rat hippocampus that subserve allocentric spatial processes: a simple problem with a complex answer. Hippocampus 10:466-474.

Amaral DG, Witter MP (1995) Hippocampal formation. In: The rat nervous system, Ed 2 (Paxinos G, ed), pp 443-493. San Diego: Academic.

Bannerman DM, Yee BK, Lemaire M, Jarrard L, Iversen SD, Rawlins JN, Good MA (2001) Contextual fear conditioning is disrupted by lesions of the subcortical, but not entorhinal, connections to the hippocampus. Exp Brain Res 141:304-311.

Blanchard RJ, Blanchard DC (1969) Crouching as an index of fear. J Comp Phys Psych 67:370-375.

Bucci DJ, Phillips RG, Burwell RD (2000) Contributions of postrhinal and perirhinal cortex to contextual information processing. Behav Neurosci 114:882-894.

Bucci DJ, Saddoris MP, Burwell RD (2002) Contextual fear discrimination is impaired by damage to postrhinal or perirhinal cortex. Behav Neurosci 116:479-488.

Burwell RD (2001) The perirhinal and postrhinal cortices of the rat: borders and cytoarchitecture. J Comp Neurol 437:17-41.

Burwell RD, Amaral DG (1998a) Perirhinal and postrhinal cortices of the rat: interconnectivity and connections with the entorhinal cortex. J Comp Neurol 391:293-321.

Burwell RD, Amaral DG (1998b) Cortical afferents of the perirhinal, postrhinal, and entorhinal cortices. J Comp Neurol 398:179-205.

Bussey TJ, Muir JL, Aggleton JP (1999) Functionally dissociating aspects of event memory: the effects of combined perirhinal and postrhinal cortex lesions on object and place memory in the rat. J Neurosci 19:495-502.

Corodimas KP, LeDoux JE (1995) Disruptive effects of posttraining perirhinal cortex lesions on conditioned fear: contributions of contextual cues. Behav Neurosci 109:613-619.

Devan BD, White NM (1999) Parallel information processing in the dorsal striatum: relation to hippocampal function. J Neurosci 19:2789-2798.

Eichenbaum H, Otto T, Cohen NJ (1994) Two functional components of the hippocampal memory system. Behav Brain Sci 17:449-518.

Fanselow MS (1980) Conditional and unconditional components of postshock freezing. Pav J Biol Sci 15:177-182.

Gallagher M, Burwell RD, Burchinal M (1993) Severity of spatial learning impairment in aging: development of a learning index for performance in the Morris water maze. Behav Neurosci 107:618-626.

Glasier MM, Janis LS, Roof RL, Stein DG (1999) Effects of unilateral entorhinal cortex lesion on retention of water maze performance. Neurobiol Learn Mem 71:19-33.

Glenn MJ, Mumby DG (1998) Place memory is intact in rats with perirhinal cortex lesions. Behav Neurosci 112:1353-1365.

Good M, Honey RC (1997) Dissociable effects of selective lesions to hippocampal subsystems on exploratory behavior, contextual learning, and spatial learning. Behav Neurosci 111:487-493.

Good M, de Hoz L, Morris RG (1998) Contingent versus incidental context processing during conditioning: dissociation after excitotoxic hippocampal plus dentate gyrus lesions. Hippocampus 8:147-159.

Hagan JJ, Verheijck EE, Spigt MH, Ruigt GS (1992) Behavioural and electrophysiological studies of entorhinal cortex lesions in the rat. Physiol Behav 51:255-266.

Herzog C, Otto T (1997) Odor-guided fear conditioning in rats: 2. Lesions of the anterior perirhinal cortex disrupt fear conditioned to the explicit conditioned stimulus but not to the training context. J Neurosci 111:1265-1272.

Herzog C, Otto T (1998) Contributions of anterior perirhinal cortex to olfactory and contextual fear conditioning. NeuroReport 9:1855-1859. 
Holland PC, Bouton ME (1999) Hippocampus and context in classical conditioning. Curr Opin Neurobiol 9:195-202.

Jarrard LE (2002) Use of excitotoxins to lesion the hippocampus: update. Hippocampus 12:405-414.

Jarrard LE, Davidson TL, Bowring B (2004)Functional differentiation within the medial temporal lobe in the rat. Hippocampus, in press.

Kaut KP, Bunsey MD (2001) The effects of lesions to the rat hippocampus or rhinal cortex on olfactory and spatial memory: retrograde and anterograde findings. Cogn Affect Behav Neurosci 1:270-286.

Knierim JJ, Kudrimoti HS, McNaughton BL (1998) Interactions between idiothetic cues and external landmarks in the control of place cells and head direction cells. J Neurophysiol 80:425-446.

Kosel KC, Van Hoesen GW, Rosene D (1983) A direct projection from the perirhinal cortex (area 35) to the subiculum in the rat. Brain Res 269:347-351.

Liu P, Bilkey D (1998) Perirhinal cortex contributions to performance in the Morris water maze. Behav Neurosci 112:304-315.

Liu P, Bilkey DK (2001) The effect of excitotoxic lesions centered on the hippocampus or perirhinal cortex in object recognition and spatial memory tasks. Behav Neurosci 115:94-111.

Liu P, Bilkey DK (2002) The effects of NMDA lesions centered on the postrhinal cortex on spatial memory tasks in the rat. Behav Neurosci $116: 860-873$

Maren S, Fanselow MS (1997) Electrolytic lesions of the fimbria/fornix, dorsal hippocampus, or entorhinal cortex produce anterograde deficits in contextual fear conditioning in rats. Neurobiol Learn Mem 67:142-149.

Maren S, Aharonov G, Fanselow MS (1997) Neurotoxic lesions of the dorsal hippocampus and Pavlovian fear conditioning in rats. Behav Brain Res $88: 261-274$

Maren S, Anagnostaras SG, Fanselow MS (1998) The startled seahorse: is the hippocampus necessary for contextual fear conditioning? Trends Cogn Sci 2:39-42.

McNish KA, Gewirtz JC, Davis M (2000) Disruption of contextual freezing, but not contextual blocking of fear-potentiated startle, after lesions of the dorsal hippocampus. Behav Neurosci 114:64-76.

Morris R (1984) Development of a water-maze procedure for studying spatial learning in the rat. J Neurosci Methods 11:47-60.

Morris RGM, Garrud P, Rawlins JNP, O'Keefe J (1982) Place navigation impaired in rats with hippocampal lesions. Nature 297:681-683.

Muller RU, Poucet B, Fenton AA, Cressant A (1999) Is the hippocampus of the rat part of a specialized navigational system? Hippocampus 9:413-422.

Mumby DG, Glenn MJ (2000) Anterograde and retrograde memory for object discriminations and places in rats with perirhinal cortex lesions. Behav Brain Res 114:119-134.

Naber PA, Caballero-Bleda M, Jorritsma-Byham B, Witter MP (1997) Parallel input to the hippocampal memory system through peri- and postrhinal cortices. NeuroReport 8:2617-2621.

Nadel L, Willner J (1980) Context and conditioning: a place for space. Phys Psych 8:218-228.

Nagahara AH, Otto T, Gallagher M (1995) Entorhinal-perirhinal lesions impair performance of rats on two versions of place learning in the Morris water maze. Behav Neurosci 109:3-9.
O'Keefe JA, Nadel L (1978) The hippocampus as a cognitive map. Oxford: Clarendon.

Oswald CJ, Good M (2000) The effects of combined lesions of the subicular complex and the entorhinal cortex on two forms of spatial navigation in the water maze. Behav Neurosci 114:211-217.

Phillips RG, LeDoux JE (1995) Lesions of the fornix but not the entorhinal or perirhinal cortex interfere with contextual fear conditioning. J Neurosci 15:5308-5315.

Pouzet B, Welzl H, Gubler MK, Broersen L, Veenman CL, Feldon J, Rawlins JN, Yee BK (1999) The effects of NMDA-induced retrohippocampal lesions on performance of four spatial memory tasks known to be sensitive to hippocampal damage in the rat. Eur J Neurosci 11:123-140.

Rapp PR, Rosenberg RA, Gallagher M (1987) An evaluation of spatial information processing in aged rats. Behav Neurosci 101:3-12.

Rudy JW, Sutherland RJ (1995) Configural association theory and the hippocampal formation: an appraisal and reconfiguration. Hippocampus 5:375-389.

Sacchetti B, Lorenzini CA, Baldi E, Tassoni G, Bucherelli C (1999) Auditory thalamus, dorsal hippocampus, basolateral amygdala, and perirhinal cortex role in the consolidation of conditioned freezing to context and to acoustic conditioned stimulus in the rat. J Neurosci 19:9570-9578.

Sacchetti B, Baldi E, Lorenzini CA, Bucherelli C (2002) Differential contribution of some cortical sites to the formation of memory traces supporting fear conditioning. Exp Brain Res 146:223-232.

Schenk F, Morris RG (1985) Dissociation between components of spatial memory in rats after recovery from the effects of retrohippocampal lesions. Exp Brain Res 58:11-28.

Shi CJ, Cassell MD (1999) Perirhinal cortex projections to the amygdaloid complex and hippocampal formation in the rat. J Comp Neurol 406:299-328.

Skelton RW, McNamara RK (1992) Bilateral knife cuts to the perforant path disrupt spatial learning in the Morris water maze. Hippocampus 2:73-80.

Stubley-Weatherly L, Harding JW, Wright JW (1996) Effects of discrete kainic acid-induced hippocampal lesions on spatial and contextual learning and memory in rats. Brain Res 716:29-38.

Tassoni G, Lorenzini CA, Baldi E, Sacchetti B, Bucherelli C (1999) A peculiar pattern of temporal involvement of rat perirhinal cortex in memory processing. Behav Neurosci 113:1161-1169.

Taube JS (1998) Head direction cells and the neurophysiological basis for a sense of direction. Prog Neurobiol 55:225-256.

Wiig KA, Bilkey DK (1994a) Perirhinal cortex lesions in rats disrupt performance in a spatial DNMS task. NeuroReport 5:1405-1408.

Wiig KA, Bilkey DK (1994b) The effects of perirhinal cortical lesions on spatial reference memory in the rat. Behav Brain Res 63:101-109.

Young SL, Bohenek DL, Fanselow MS (1994) NMDA processes mediate anterograde amnesia of contextual fear conditioning induced by hippocampal damage: immunization against amnesia by context preexposure. Behav Neurosci 108:19-29.

Zola-Morgan S, Squire LR, Ramus SJ (1994) Severity of memory impairment in monkeys as a function of locus and extent of damage within the medial temporal lobe of memory system. Hippocampus 4:483-495. 